\title{
Podocyte dedifferentiation: a specialized process for a specialized cell
}

\section{Carl James May*, Moin Saleem and Gavin lain Welsh}

Academic Renal Unit, University of Bristol, Bristol, UK

\section{Edited by:}

Maciej Wojciech Jankowski, Medical

University of Gdansk, Poland

\section{Reviewed by:}

Elena Torban, McGill University,

Canada

David Andrew Long, University

College London, UK

\section{*Correspondence:}

Carl James May, Academic Renal Unit, University of Bristol, Dorothy Hodgkin Building, Whitson Street, Bristol BS1 3NY, UK

e-mail:carl.may@bristol.ac.uk

\begin{abstract}
The podocyte is one of the two cell types that contribute to the formation of the glomerular filtration barrier (GFB). It is a highly specialized cell with a unique structure. The key feature of the podocyte is its foot processes that regularly interdigitate. A structure known as the slit diaphragm can be found bridging the interdigitations. This molecular sieve comprises the final layer of the GFB. It is well accepted that the podocyte is the target cell in the pathogenesis of nephrotic syndrome. In nephrotic syndrome, the GFB no longer restricts the passage of macromolecules and protein is lost into the urine. A number of phenotypic and morphological changes are seen in the diseased podocyte and in the literature these have been described as an epithelial-mesenchymal transition (EMT). However, there is a growing appreciation that this term does not accurately describe the changes that are seen. Definitions of type-2 EMT are based on typical epithelial cells. While the podocyte is known as a visceral epithelial cell, it is not a typical epithelial cell. Moreover, podocytes have several features that are more consistent with mesenchymal cells. Therefore, we suggest that the term podocyte disease transformation is more appropriate.
\end{abstract}

Keywords: nephrotic syndrome, podocytes, proteinuria, epithelial-mesenchymal transition, dedifferentiation

\section{INTRODUCTION}

The podocyte is thought to be the target cell in the pathogenesis of nephrotic syndrome. The term "podocytopathy" is being increasingly used to describe disease that has arisen due to insult or injury to the podocyte. Minimal change nephropathy (MCN), focal segmental glomerulosclerosis (FSGS), diffuse mesangial sclerosis (DMS), and collapsing glomerulopathy (CG) are all thought to be podocytopathic nephrotic diseases (1). Irrespective of the cause, podocytes demonstrate dramatic morphological differences when there is nephrotic range proteinuria (2). Actin cytoskeleton rearrangement, slit diaphragm loss, and more cuboidal morphology are all hallmark features of the diseased podocyte (2). The field has tended to refer to this loss of typical phenotype as an epithelial-mesenchymal transition (EMT) event (3-5).

However, EMT is a highly plastic and reversible process: indeed, the word "transition" was chosen deliberately to reflect this transient nature. The phenotypic and morphological changes seen in diseased podocytes are only reversible if the insult is not very severe. In minimal change disease, the disease phenotype is reversible following glucocorticoid therapy. However, in FSGS, the changes in morphology and phenotype are not only irreversible but progressive (6). Therefore, using the term EMT implies an innate reversibility, which is not strictly accurate in the case of the podocyte. Therefore, it is important to reassess the extent to which the term EMT accurately describes the transposition from the healthy to the diseased podocyte morphology. Additionally, the podocyte is not a typical epithelial cell. Despite podocytes also being known as visceral epithelial cells, they retain several mesenchymal features (spindle shaped morphology and high levels of matrix interaction) and lack archetypal epithelial markers (cell-cell contacts that are based predominantly on P rather than
E-cadherin). Therefore, the podocyte fails to demonstrate the emblematic features of EMT. If the change in podocyte morphology and phenotype seen in disease does not fulfill the accepted criteria for an EMT event and is not necessarily transient in nature, then the usefulness of this term in this context must be questioned.

Immortalized podocytes in vitro respond to the classic inducer of EMT, TGF-B1. Following TGF-B1 treatment, human podocytes in vitro demonstrate increased levels of $\alpha$-SMA, cadherin switch from P-cadherin to $\mathrm{N}$-cadherin, and expression of the main effector transcription factors of EMT: SNAIL and SLUG (7). Similar phenotypic changes are seen in mouse podocytes in vitro when exposed to TGF-B1. Again suppression of P-cadherin along with suppression of ZO-1 and nephrin with concomitant upregulation of desmin, fibronectin, and collagen I is observed (8). In vitro, following $24 \mathrm{~h}$ exposure to TGF-B1, human podocytes lose their highly arborized morphology and adopt a more cobblestone-like morphology. The existence of similar phenotypic changes in both mouse and human podocytes in response to TGF-B1 is indicative of an evolutionarily conserved disease mechanism.

The pathological effects of such phenotypic changes within a cell-type, such as the podocyte, are severe. Foot process effacement is linked to a diminished ability to restrict urinary protein loss. This leads to runaway proteinuria and nephrotic syndrome. Effaced podocytes have less contact with the glomerular basement membrane, making podocyte loss much more likely. Additionally, the increased synthesis of extracellular matrix (ECM) components such as fibronectin and collagen I may contribute to GBM thickening. These are all hallmark features of diabetic nephropathy (9).

FSP1 is a fibroblast (mesenchymal) marker, which has been found in podocytes from patients with diabetic nephropathy. Healthy podocytes do not express FSP1; therefore, de novo 
expression of a mesenchymal marker is a pathological change in phenotype. Moreover, the frequency of $\mathrm{FSP}^{+}$podocytes in the urine has been linked to disease severity (8). FSP1 $1^{+}$podocytes have also been found in the glomeruli of FSGS patients (10).

The term EMT is over simplistic in this context, and does not encapsulate the process that is seen in the disease state in vivo and disease models in vitro. Broadly speaking, the morphological and phenotypic changes seen in diseased podocytes appear to be an EMT type event. However, in order for the assertion that this is an EMT event to be accurate, one must first consider to what extent the changes seen correlate with the definition of EMT.

\section{THE PODOCYTE: A SPECIALIZED CELL}

The podocyte is a highly specialized cell that is situated on the outer surface of the GBM. It comprises three structurally and functionally unique segments. These are known as the cell body, the major process, and the foot process (11). The cell body, the major processes, and the foot processes share a common actin cytoskeleton contractile apparatus similar to that found in smooth muscle cells or pericytes (11). The foot processes extend from the major processes and cover the GBM. Neighboring foot processes interdigitate, and where this occurs a modified cellto-cell junction known as the slit diaphragm is formed (12). The slit diaphragm forms the final layer of the glomerular filtration barrier (GFB) and has both charge and size selective properties (13).

The foot processes are the main functional unit of the podocyte. These contain loops of filamentous actin (F-actin) that can be assembled, disassembled, and bundled together in response to the changing requirements of the foot process. The tensile strength of F-actin and its concentration in the foot processes enable the podocyte to withstand the pressure of glomerular flow (14). It is this ability of actin, to be soluble as a monomer and then rapidly polymerize to provide structure and support that allows the redistribution of the podocytes foot processes. Bivalent molecules, such as $\alpha$-actinin- 4 and dystrophin can link bundled actin fibers for added strength (15). There are more than 100 proteins that are involved in the regulation of actin filament formation and breakdown indicating that these are essential procedures (16). The importance of the actin cytoskeletal regulation in the proper functioning of the podocyte is indicated by the number of socalled "nephrotic" genes whose protein products act on the actin cytoskeleton (17) (Table S1 in the Supplementary Material).

Such is the importance of the podocyte and the slit diaphragm in particular that there are multiple monogenic mutations that cause nephrotic syndrome as shown in Table S1 of Supplementary Material.

Structural and functional defects in the GFB result in an inability to restrict urinary protein loss. Nephrotic syndrome is defined by the triad of proteinuria, hypoalbuminemia, and edema. Proteinuria is defined by presence of non-physiological levels of a mixture of proteins in the urine (>200 mg/l) (20). However, in clinical practice, the albumin-creatinine ratio is more likely to be employed as it accounts for differences in urine dilution, i.e., a level of $>30 \mu \mathrm{g} / \mathrm{mg}$ demonstrates proteinuria (21). Structural and functional defects in the GFB result in an inability to restrict urinary protein loss.
Podocytes are terminally differentiated cells meaning that they are unable to proliferate. This lack of podocyte proliferation limits their capacity to recover from any damage. Therefore, podocyte injury is thought to be central to nephrotic syndrome pathogenesis. The range of podocyte injuries that can play a role in nephrotic syndrome pathogenesis is collectively referred to as podocytopathies. Many of these podocytopathies are caused by mutations in key genes. These genes are central to podocyte function; encoding either slit diaphragm proteins, transcription factors, or signaling mediators. The genetic causes of nephrotic syndrome are discussed in detail in an excellent review by Hildebrandt (17). In addition to genetic podocytopathies, there are also reactive podocytopathies. In these reactive podocytopathies, the podocyte is damaged by mediators in the microenvironment (1). These extrinsic podocyte stressors can be from several sources. They can be viral, toxic, immune-mediated, mechanical, or metabolic disorder derived.

\section{EPITHELIAL-MESENCHYMAL TRANSITION}

It is well accepted that a loss of the highly specialized podocyte structure limits the capacity of the final layer of the GFB to restrict urinary protein loss. The changes seen in diseased podocytes could be due to an EMT event.

It has been posited in the literature that since podocytes develop from mesenchymal cells via a mesenchymal-epithelialtransition (MET) event, it is reasonable to assume that the loss of mature podocyte characteristics is the reverse of this process (22). Glomerular development consists of four stages as follows: the vesicle stage, the $S$-shaped body stage, the capillary loop stage, and the maturation stage (23). The early podocytes can first be distinguished as a layer of cells at the proximal end of the $S$-shaped body. During this stage, the podocytes develop from the columnar epithelial cells (24). In turn, the columnar epithelial cells arise from the metanephric mesenchyme via a MET event (25). At this point, the podocytes express specific podocyte markers such as Wilms' tumor suppressor (WT1) and nephrin $(26,27)$. As the capillary loops form, the podocyte progenitors lose their lateral cell-cell contacts and begin to migrate. As the capillary loop stage progresses, the podocyte foot processes form. At this point, expression of the slit diaphragm proteins, nephrin, podocin, and CD2AP can be seen (28-30).

Epithelial-to-mesenchymal transition is a tightly regulated process by which epithelial cells lose their hallmark epithelial characteristics and gain the features of mesenchymal cells. EMT can be initiated in response to circulating mediators such as TGF-B1 (31, 32). During the process of EMT, the podocytes should lose their epithelial polarity, the cell-to-cell junctions (the slit diaphragm) will be altered, and the actin cytoskeleton will be rearranged (33). Following stimulation with TGF-B1, podocytes lose expression of nephrin and ZO-1 (34). As important slit diaphragm proteins, the loss of nephrin and ZO-1 expression is detrimental to the function of the podocyte. Not only does TGF-B1 lead to loss of epithelial characteristics in podocytes (as evidenced by the loss of nephrin expression) but also an increase in mesenchymal characteristics. Desmin is one such mesenchymal marker that is upregulated by TGF-B1, moreover, desmin upregulation by podocytes is seen in glomerular diseases where podocyte damage is a key feature (35). 
These changes have severe consequences for the structure and function of the podocyte cell. The loss of epithelial cell-cell junctions during EMT is best represented by a loss of the slit diaphragm in the diseased podocyte. As previously described, podocytes demonstrate a clear change in their morphology and phenotype in the diseased state. Expression of the slit diaphragm is lost and the foot processes are effaced. However, does the loss of essential podocyte features seen in disease and disease models, both in vivo and in vitro, respectively, represent a typical EMT event?

Epithelial-mesenchymal transition has been categorized into three types: type 1, associated with implantation, embryo formation, and organ development, type 2, associated with wound healing tissue regeneration and organ fibrosis, and type 3, which is associated with cancer metastasis and progression (36). The distinctions between these subtypes of EMT are outside the scope of this review but are extensively covered in a review by Kalluri and Weinberg (36). For the purposes of this review, EMT will now be a reference to type-2 EMT. The criteria for type-2 EMT are listed in Table 1.

The changes in morphology and phenotype between healthy and diseased podocytes only partially satisfies most of the criteria for type-2 EMT and does not fit at all with the rest. The table demonstrates quite clearly the inaccuracies of describing this process as EMT. For instance, one of the criteria for type-2 EMT is a cadherin switch from $\mathrm{E}$-cadherin to $\mathrm{N}$-cadherin. While the podocytes do undergo a cadherin switch in the diseased state, this is from $\mathrm{P}$-cadherin to $\mathrm{N}$-cadherin. No evidence could be found in the literature for podocytes expressing DDR2 or HSP47. Neither there is clear evidence for the nuclear translocation of TWIST. Otherwise, it is the lack of typical epithelial phenotype in podocytes that precludes them from matching this definition of EMT.

First of all, podocytes are atypical epithelial cells. While it is the case that podocytes demonstrate epithelial features, such as clear apical-basal cell polarity, they also exhibit mesenchymal features such as vimentin and intermediate filament expression (16). They also express features of differentiated mesenchymal cells, in particular smooth muscle actin, akin to a pericyte phenotype (42).

High migration capacity is a mesenchymal feature, while low invasive capacity is an epithelial feature, again demonstrating the dichotomous nature of the podocyte. The healthy podocyte maintains a dynamic range within which motility is regulated (43). It has been shown that plasma from patients with active FSGS significantly increases podocyte motility in vitro (44). The insult caused by the disease is increasing the mesenchymal characteristic of the podocyte. These hypermotile podocytes are not invasive due to the location of the podocytes on the outside of the GBM. The flux of hyperfiltrate through the GFB leads to a loss of podocytes in the urine. Indeed, as mentioned previously, viable urinary podocytes have been found with mesenchymal fibroblastic markers such as FSP1 (25).

\section{Table 1 | Hallmark characteristics of EMT.}

\begin{tabular}{|c|c|c|}
\hline Criteria & Evidence in the literature & Criteria met? \\
\hline $\begin{array}{l}\text { Novel FSP1 and DDR2 } \\
\text { expression associated with } \\
\text { basement membrane } \\
\text { disruption }\end{array}$ & $\begin{array}{l}\text { When podocytes are exposed to high glucose concentration in vitro, they demonstrate a clear } \\
\text { upregulation of FSP1 (37). Urinary podocytes from diabetic nephropathy patients are FSP1+ (5). } \\
\text { Moreover, ectopic overexpression of the known EMT inducer TGF-B1 in the glomerulus stimulates } \\
\text { FSP1 in the podocyte (38) }\end{array}$ & Partially \\
\hline $\begin{array}{l}\text { Increased expression of } \\
\text { HSP47, collagen } 1(\alpha 1) \text {, } \\
\text { collagen } 2(\alpha 2) \text {, or vimentin }\end{array}$ & $\begin{array}{l}\text { HSP47 is a marker of collagen producing cells and has been found in crescentic cells but not in } \\
\text { podocytes in vivo or in vitro. TGF-B1, a potent EMT inducer stimulates collagen } 1 \text { expression in mouse } \\
\text { podocytes (8). Although mature podocytes express vimentin, the expression increases following } \\
\text { TGF-B1 treatment in vitro (39) }\end{array}$ & Partially \\
\hline $\begin{array}{l}\text { Cadherin switch from } \\
\text { E-cadherin to N-cadherin }\end{array}$ & $\begin{array}{l}\text { The typical switch from E- to } \mathrm{N} \text {-cadherin expression is not seen since mature podocytes do not } \\
\text { express E-cadherin (40). They do, however, express P-cadherin. A switch from P-cadherin to N-cadherin } \\
\text { is seen following TGF-B1 treatment (7) }\end{array}$ & No \\
\hline $\begin{array}{l}\text { Nuclear relocalization of } \\
\text { CBF-A or B-catenin or new } \\
\text { expression of SNAIL, SLUG, } \\
\text { or TWIST }\end{array}$ & $\begin{array}{l}\text { Nuclear translocation of beta-catenin is seen in experimental models of nephrotic syndrome both } \\
\text { in vitro and in vivo and also in diabetic nephropathy (41). Wnt signaling is responsible for the } \\
\text { translocation of beta-catenin and plays an important role in podocyte injury and proteinuria (41). TGF-B1 } \\
\text { treatment stimulates SNAIL expression in vitro. Additionally, ectopic expression of SNAIL induces } \\
\text { changes in podocyte phenotype consistent with EMT (8). SLUG is also expressed following TGF-B1 } \\
\text { treatment in vitro (7) }\end{array}$ & Partially \\
\hline $\begin{array}{l}\text { Loss or reduction of epithelial } \\
\text { cell markers }\end{array}$ & $\begin{array}{l}\text { The podocyte dedifferentiation seen in response to TGF-B1 treatment is associated with a reduction in } \\
\text { epithelial markers such as ZO-1 and P-cadherin }(8,39)\end{array}$ & Partially \\
\hline $\begin{array}{l}\text { Spindle shape morphology } \\
\text { with redistribution of stress } \\
\text { fibers and loss of polarity }\end{array}$ & $\begin{array}{l}\text { Podocytes have a spindle-like arborized morphology when fully differentiated. This morphology is lost } \\
\text { following insult. A loss of apical-basal polarity leads to the mislocalization of nephrin and concomitant } \\
\text { proteinuria. This loss of polarity has not been seen in models of EMT either in vitro or in vivo }\end{array}$ & No \\
\hline
\end{tabular}

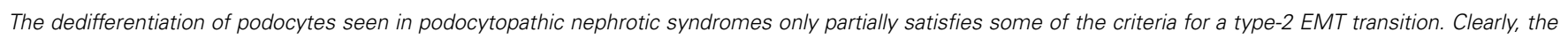
morphological and phenotypic changes seen in diseased podocytes are not obtained via a type-2 EMT event. 
Podocytes express P-cadherin instead of E-cadherin; again this is atypical for an epithelial cell $(7,45)$. The generation of a spindle-like morphology is indicative of a mesenchymal phenotype. However, normal podocytes both in vitro and in vivo have a highly arborized structure that is consistent with the aforementioned spindle-like morphology. Following dedifferentiation, the podocyte actin cytoskeleton rearrangement causes foot process effacement leading to the morphology seen in Figure 1. This effaced morphology could be described as analogous to the typical epithelial "cobblestone" morphology. In this way, following dedifferentiation, podocyte morphology goes from the mesenchymal-like highly arborized morphology to the epithelial-like "cobblestone" morphology. The epithelial and mesenchymal characteristics of the podocyte are summarized in Figure 1.

Therefore, when describing podocyte dedifferentiation, one must be careful to consider to what differential state the podocyte is reverting. A partial reversal of the mature, partially mesenchymal state to a more immature epithelial state seen during the $S$-shaped body, and capillary loop stages of development could be termed as MET. However, complete dedifferentiation to the "mesenchymal rest" state of development could also conceivably occur, and be more accurately described as EMT, though in neither case is the terminology adequately nuanced.

The terminology "EMT" was adapted from the original term "epithelial-mesenchymal transformation" in order to emphasize the transient nature of this mechanism and the potential for its reversal (46). Clearly, the podocyte is not the average epithelial cell and its dedifferentiation does not entirely fit with the definition of EMT outlined in this article. This is more than simply a semantic argument. Evidently, mediators such as TGF-B1 can instigate EMT and induce podocyte dedifferentiation that is at least reminiscent of EMT. However, by constraining the conceptualization of this process by only thinking in terms of EMT, novel mediators and disease processes causing podocyte dedifferentiation may be missed. In light of the criticisms of this term to describe podocyte dedifferentiation in disease as EMT, perhaps the term podocyte disease transformation (PDT) is more appropriate. With transformation reflecting that PDT is less transient than EMT.

\section{GENETIC FORMS OF NEPHROTIC SYNDROME AND EMT}

Single gene defects causing NS tend to cause irreversible podocyte damage. This is to be expected since many of the genes that are known to cause nephrotic syndrome express proteins that are key components of the slit diaphragm (nephrin and podocin) or are essential to the specialized architecture of the podocyte (such as alpha actinin IV). However, the incorporation of transition in this term is deliberate. The diseased podocyte phenotype can be transient. Nephrotic syndrome is often treated with glucocorticoids and/or calcineurin inhibitors both of which have been shown to have direct effects on the actin cytoskeleton of the podocyte (47-49). One key renal developmental gene, known to cause NS when mutated, has been shown to result in features of podocyte dedifferentiation. This is WT1, a transcription factor both highly expressed and necessary for renal development (50), yet only expressed in the podocyte in the mature kidney. Mouse models of the human disease resulting from WT1 mutations,
Denys-Drash Syndrome (DDS), reveal loss of podocyte ZO-1 expression and upregulation of podocyte TGF-B (51). Furthermore, loss of WT1 and re-expression of PAX2 and cytokeratin in podocytes have been described in cellular lesions in FSGS biopsies, implying an epithelial switch during acquired disease (52). Our own data examining the phenotype of conditionally immortalized podocytes from children with DDS confirms re-expression of PAX2, but also clear mesenchymal features suggestive of complete dedifferentiation (unpublished results). Thus, WT1 may be a key regulator of podocyte EMT both developmentally and as a target in disease.

The extracellular domain of nephrin forms the protein scaffold of the slit diaphragm (53). There are eight IgG-like motifs in the extracellular domain each of which contains two cysteine residues that are bound to each other via disulfide bridges. In addition, there are three "free" cysteine residues per nephrin molecule that are available to form disulfide bridges with bordering nephrin molecules (54). In this way, nephrin forms the scaffold for the slit diaphragm. The extracellular domain of nephrin interacts with other proteins in order to maintain the integrity of the barrier. Two such proteins are Neph 1 and Neph 2 (55). Clearly, the loss of most of the extracellular domain, as in patients with the Finmajor mutation, has massive implications for the formation of the slit diaphragm (56). The cytoplasmic domain of nephrin, of which part is missing in patients with the Fin-minor mutation, plays a role in the maintenance of the structural and functional capabilities of nephrin (56). The cytoplasmic domain also connects nephrin, and hence the slit diaphragm to the actin cytoskeleton of the foot process (57). It has been suggested that nephrin can bind directly to the actin cytoskeleton of the foot process via its cytoplasmic domain, while Yuan and colleagues have shown that nephrin is at least capable of binding to actin (58).

Podocin comprises 383 amino acids and has a hairpin structure such that both the $\mathrm{C}$ and $\mathrm{N}$ terminus are cytoplasmic (59). It is a raft-associated constituent of the foot process membrane that is localized at the insertion of the slit diaphragm itself (29). Within the raft, podocin can form oligomers, which lead to invagination of the foot process membrane, to which CD2AP and nephrin are recruited (60). In fact, a fully functioning podocin is required for nephrin transport to the membrane (61). This is the foundation of the slit diaphragm assembly (62). Between 10 and $28 \%$ of all nonfamilial childhood, SRNS cases are caused by recessive podocin mutations, such is the importance of this protein (62).

$\mathrm{CD} 2 \mathrm{AP}$ is an adaptor molecule that possesses a coiled coil domain and 3 Src homology 3 (SH3) domains $(59,63)$. It also has an actin binding site at its $\mathrm{NH}_{2}$ terminus and is believed to contribute to dynamic actin assembly $(64,65)$. CD2AP is capable of interacting with nephrin and in complex with nephrin and podocin is able to recruit PI3K to the plasma membrane (63).

\section{CONCLUSION}

In reactive podocytopathies, the podocyte is injured by a circulating factor (66). It is clear, however, that the podocytes undergo a set of phenotypic and morphological changes during nephrotic syndrome. This process has been likened to EMT. TGF-B1 is a potent inducer of EMT. The research described in this review has relied heavily on in vitro work centered on the response of 


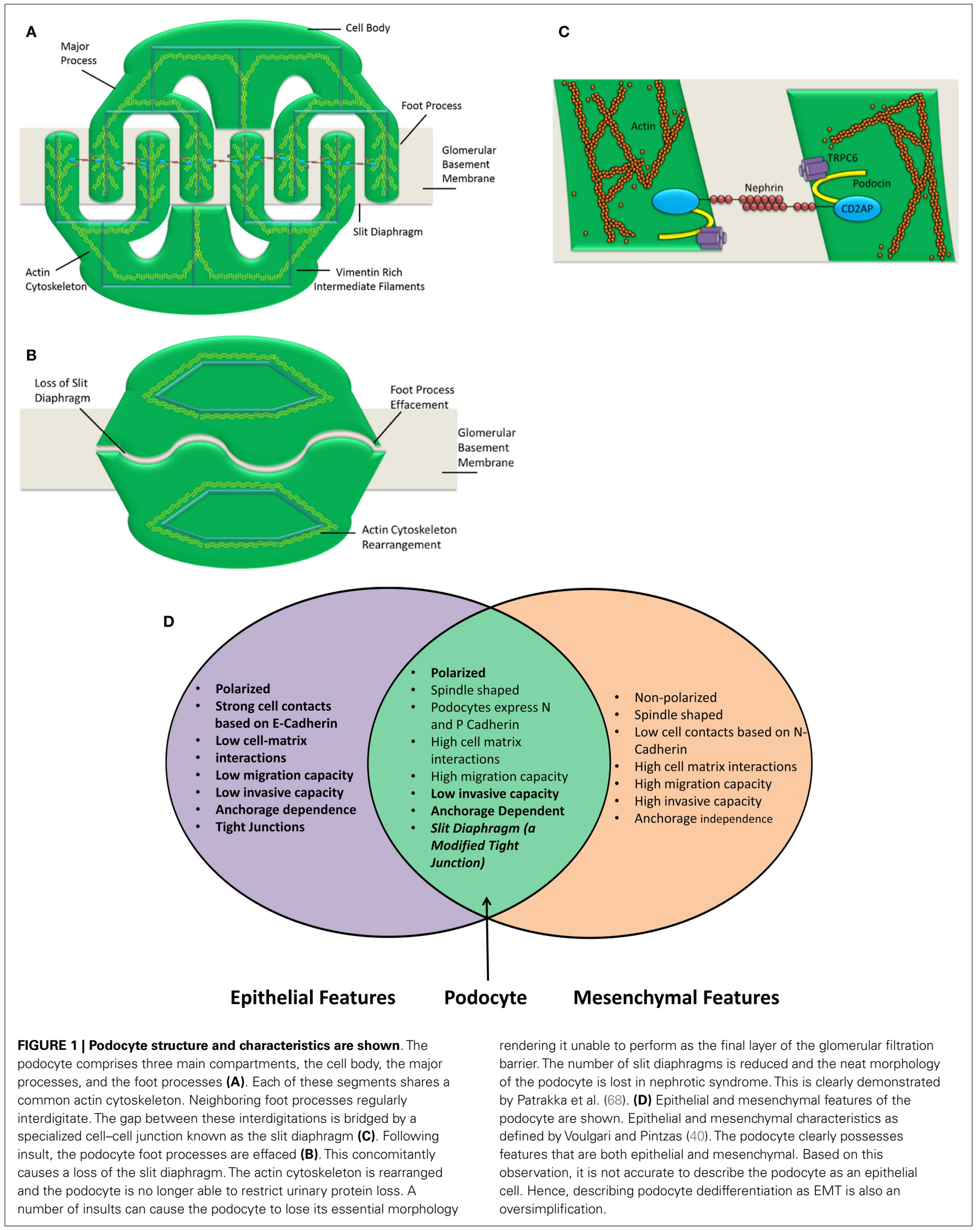


podocytes to TGF-B1 treatment. As demonstrated, the podocytes do not undergo a typical type-2 EMT in response to TGF-B1. The new term "podocyte disease transformation" has been coined in order to distinguish this process from EMT.

A new technique known as transcriptome in vivo analysis (TIVA), has been developed to analyze the transcriptome of single cell populations in vivo without destroying the tissue (67). This technique could be employed to further study the changes in podocytes in disease models.

The traditional definition of a podocyte as an epithelial cell is clearly simplistic, and in fact, this is a uniquely differentiated cell, fit for a specific functional purpose that fulfills features of a partial mesenchymal and partial epithelial cell. It reaches this mature phenotype via immature mesenchymal and then epithelial stages, and therefore, dedifferentiation in disease could result in regression to either of these states. It will be important in understanding podocyte disease to understand the drivers of these changes, and recognizing the developmental features that are correlated with specific clinical conditions.

\section{SUPPLEMENTARY MATERIAL}

The Supplementary Material for this article can be found online at http://www.frontiersin.org/Journal/10.3389/fendo.2014.00148/ abstract

\section{REFERENCES}

1. Barisoni L, Schnaper HW, Kopp JB. A proposed taxonomy for the podocytopathies: a reassessment of the primary nephrotic diseases. Clin J Am Soc Nephrol (2007) 2(3):529-42. doi:10.2215/CJN.04121206

2. Sedor JR, Madhavan SM, Kim JH, Konieczkowski M. Out on a LIM: chronic kidney disease, podocyte phenotype and the Wilm's tumor interacting protein (WTIP). Trans Am Clin Climatol Assoc (2011) 122:184-97.

3. Liu Y. New insights into epithelial-mesenchymal transition in kidney fibrosis. J Am Soc Nephrol (2010) 21(2):212-22. doi:10.1681/ASN.2008121226

4. Lee HS. Mechanisms and consequences of TGF-ss overexpression by podocytes in progressive podocyte disease. Cell Tissue Res (2012) 347(1):129-40. doi:10. 1007/s00441-011-1169-7

5. Yamaguchi Y, Iwano M, Suzuki D, Nakatani K, Kimura K, Harada K, et al. Epithelial-mesenchymal transition as a potential explanation for podocyte depletion in diabetic nephropathy. Am J Kidney Dis (2009) 54(4):653-64. doi:10.1053/j.ajkd.2009.05.009

6. D’Agati VD, Kaskel FJ, Falk RJ. Focal segmental glomerulosclerosis. N Engl J Med (2011) 365(25):2398-411. doi:10.1056/NEJMral106556

7. Ghiggeri GM, Gigante M, Di Donato A. Constitutional nephrin deficiency in conditionally immortalized human podocytes induced epithelial-mesenchymal transition, supported by beta-catenin/nf-kappa b activation: a consequence of cell junction impairment? Int J Nephrol (2013) 2013:457490. doi:10.1155/2013/ 457490

8. Li Y, Kang YS, Dai C, Kiss LP, Wen X, Liu Y. Epithelial-to-mesenchymal transition is a potential pathway leading to podocyte dysfunction and proteinuria. Am J Pathol (2008) 172(2):299-308. doi:10.2353/ajpath.2008.070057

9. Jefferson JA, Shankland SJ, Pichler RH. Proteinuria in diabetic kidney disease: a mechanistic viewpoint. Kidney Int (2008) 74(1):22-36. doi:10.1038/ki. 2008.128

10. Samejima K, Nakatani K, Suzuki D, Asai O, Sakan H, Yoshimoto S, et al. Clinical significance of fibroblast-specific protein-1 expression on podocytes in patients with focal segmental glomerulosclerosis. Nephron Clin Pract (2012) 120(1):c1-7. doi: $10.1159 / 000334184$

11. Faul C, Asanuma K, Yanagida-Asanuma E, Kim K, Mundel P. Actin up: regulation of podocyte structure and function by components of the actin cytoskeleton. Trends Cell Biol (2007) 17(9):428-37. doi:10.1016/j.tcb.2007.06.006

12. Mundel P, Shankland SJ. Podocyte biology and response to injury. J Am Soc Nephrol (2002) 13(12):3005-15. doi:10.1097/01.ASN.0000039661.06947.FD
13. Tryggvason K. Unraveling the mechanisms of glomerular ultrafiltration: nephrin, a key component of the slit diaphragm. J Am Soc Nephrol (1999) 10(11):2440-5.

14. Pavenstadt H, Kriz W, Kretzler M. Cell biology of the glomerular podocyte. Physiol Rev (2003) 83(1):253-307.

15. Gordon AM, Homsher E, Regnier M. Regulation of contraction in striated muscle. Physiol Rev (2000) 80(2):853-924.

16. Welsh GI, Saleem MA. The podocyte cytoskeleton - key to a functioning glomerulus in health and disease. Nat Rev Nephrol (2012) 8(1):14-21. doi:10.1038/nrneph.2011.151

17. Hildebrandt F. Genetic kidney diseases. Lancet (2010) 375(9722):1287-95. doi:10.1016/S0140-6736(10)60236-X

18. Brinkkoetter PT, Ising C, Benzing T. The role of the podocyte in albumin filtration. Nat Rev Nephrol (2013) 9(6):328-36. doi:10.1038/nrneph.2013.78

19. McCarthy HJ, Bierzynska A, Wherlock M, Ognjanovic M, Kerecuk L, Hegde $S$, et al. Simultaneous sequencing of 24 genes associated with steroid-resistant nephrotic syndrome. Clin J Am Soc Nephrol (2013) 8(4):637-48. doi:10.2215/ CJN.07200712

20. Nielsen R, Christensen EI. Proteinuria and events beyond the slit. Pediatr Nephrol (2010) 25(5):813-22. doi:10.1007/s00467-009-1381-9

21. Hutchison AS, O'Reilly DS, MacCuish AC. Albumin excretion rate, albumin concentration, and albumin/creatinine ratio compared for screening diabetics for slight albuminuria. Clin Chem (1988) 34(10):2019-21.

22. Little MH, McMahon AP. Mammalian kidney development: principles, progress, and projections. Cold Spring Harb Perspect Biol (2012) 4(5). doi:10.1101/ cshperspect.a008300

23. Takano K, Kawasaki Y, Imaizumi T, Matsuura H, Nozawa R, Tannji M, et al. Development of glomerular endothelial cells, podocytes and mesangial cells in the human fetus and infant. Tohoku J Exp Med (2007) 212(1):81-90. doi:10.1620/tjem.212.81

24. Kreidberg JA. Podocyte differentiation and glomerulogenesis. J Am Soc Nephrol (2003) 14(3):806-14. doi:10.1097/01.ASN.0000054887.42550.14

25. Reidy K, Susztak K. Epithelial-mesenchymal transition and podocyte loss in diabetic kidney disease. Am J Kidney Dis (2009) 54(4):590-3. doi:10.1053/j.ajkd. 2009.07.003

26. Armstrong JF, Pritchard-Jones K, Bickmore WA, Hastie ND, Bard JB. The expression of the Wilms' tumour gene, WT1, in the developing mammalian embryo. Mech Dev (1993) 40(1-2):85-97. doi:10.1016/0925-4773(93)90090-K

27. Putaala H, Sainio K, Sariola H, Tryggvason K. Primary structure of mouse and rat nephrin cDNA and structure and expression of the mouse gene. J Am Soc Nephrol (2000) 11(6):991-1001.

28. Kawachi H, Koike H, Kurihara H, Yaoita E, Orikasa M, Shia MA, et al. Cloning of rat nephrin: expression in developing glomeruli and in proteinuric states. Kidney Int (2000) 57(5):1949-61. doi:10.1046/j.1523-1755.2000.00044.x

29. Boute N, Gribouval O, Roselli S, Benessy F, Lee H, Fuchshuber A, et al. NPHS2, encoding the glomerular protein podocin, is mutated in autosomal recessive steroid-resistant nephrotic syndrome. Nat Genet (2000) 24(4):349-54. doi: $10.1038 / 74166$

30. Li C, Ruotsalainen V, Tryggvason K, Shaw AS, Miner JH. CD2AP is expressed with nephrin in developing podocytes and is found widely in mature kidney and elsewhere. Am J Physiol Renal Physiol (2000) 279(4): F785-92.

31. Bottinger EP, Bitzer M. TGF-beta signaling in renal disease. J Am Soc Nephrol (2002) 13(10):2600-10. doi:10.1097/01.ASN.0000033611.79556.AE

32. Chanan-Khan A, Miller KC, Musial L, Padmanabhan S, Yu J, Ailawadhi S, et al. Bortezomib in combination with pegylated liposomal doxorubicin and thalidomide is an effective steroid independent salvage regimen for patients with relapsed or refractory multiple myeloma: results of a phase II clinical trial. Leuk Lymphoma (2009) 50(7):1096-101. doi:10.1080/10428190902912460

33. Valcourt U, Kowanetz M, Niimi H, Heldin CH, Moustakas A. TGF-beta and the Smad signaling pathway support transcriptomic reprogramming during epithelial-mesenchymal cell transition. Mol Biol Cell (2005) 16(4):1987-2002. doi:10.1091/mbc.E04-08-0658

34. Shankland SJ. The podocyte's response to injury: role in proteinuria and glomerulosclerosis. Kidney Int (2006) 69(12):2131-47. doi:10.1038/sj.ki. 5000410

35. Zou J, Yaoita E, Watanabe Y, Yoshida Y, Nameta M, Li H, et al. Upregulation of nestin, vimentin, and desmin in rat podocytes in response to injury. Virchows Arch (2006) 448(4):485-92. doi:10.1007/s00428-005-0134-9 
36. Kalluri R, Weinberg RA. The basics of epithelial-mesenchymal transition. J Clin Invest (2009) 119(6):1420-8. doi:10.1172/JCI39104

37. Lv Z, Hu M, Zhen J, Lin J, Wang Q, Wang R. Rac1/PAK1 signaling promotes epithelial-mesenchymal transition of podocytes in vitro via triggering betacatenin transcriptional activity under high glucose conditions. Int J Biochem Cell Biol (2013) 45(2):255-64. doi:10.1016/j.biocel.2012.11.003

38. Wang D, Dai C, Li Y, Liu Y. Canonical Wnt/beta-catenin signaling mediates transforming growth factor-beta1-driven podocyte injury and proteinuria. Kidney Int (2011) 80(11):1159-69. doi:10.1038/ki.2011.255

39. Herman-Edelstein M, Thomas MC, Thallas-Bonke V, Saleem M, Cooper ME, Kantharidis P. Dedifferentiation of immortalized human podocytes in response to transforming growth factor-beta: a model for diabetic podocytopathy. Diabetes (2011) 60(6):1779-88. doi:10.2337/db10-1110

40. Voulgari A, Pintzas A. Epithelial-mesenchymal transition in cancer metastasis: mechanisms, markers and strategies to overcome drug resistance in the clinic. Biochim Biophys Acta (2009) 1796(2):75-90. doi:10.1016/j.bbcan.2009.03.002

41. Dai C, Stolz DB, Kiss LP, Monga SP, Holzman LB, Liu Y. Wnt/beta-catenin signaling promotes podocyte dysfunction and albuminuria. J Am Soc Nephrol (2009) 20(9):1997-2008. doi:10.1681/ASN.2009010019

42. Saleem MA, Zavadil J, Bailly M, McGee K, Witherden IR, Pavenstadt H, et al. The molecular and functional phenotype of glomerular podocytes reveals key features of contractile smooth muscle cells. Am J Physiol Renal Physiol (2008) 295(4):F959-70. doi:10.1152/ajprenal.00559.2007

43. Kistler AD, Altintas MM, Reiser J. Podocyte GTPases regulate kidney filter dynamics. Kidney Int (2012) 81(11):1053-5. doi:10.1038/ki.2012.12

44. Harris JJ, McCarthy HJ, Ni L, Wherlock M, Kang H, Wetzels JF, et al. Active proteases in nephrotic plasma lead to a podocin-dependent phosphorylation of VASP in podocytes via protease activated receptor-1. J Pathol (2013) 229(5):660-71. doi:10.1002/path.4149

45. Lehtonen S, Lehtonen E, Kudlicka K, Holthöfer H, Farquhar MG. Nephrin forms a complex with adherens junction proteins and CASK in podocytes and in Madin-Darby canine kidney cells expressing nephrin. Am J Pathol (2004) 165(3):923-36. doi:10.1016/S0002-9440(10)63354-8

46. Lamouille S, Xu J, Derynck R. Molecular mechanisms of epithelial-mesenchymal transition. Nat Rev Mol Cell Biol (2014) 15(3):178-96. doi:10.1038/nrm3758

47. Ransom RF, Lam NG, Hallett MA, Atkinson SJ, Smoyer WE. Glucocorticoids protect and enhance recovery of cultured murine podocytes via actin filament stabilization. Kidney Int (2005) 68(6):2473-83. doi:10.1111/j.1523-1755.2005. 00723.x

48. Xing CY, Saleem MA, Coward RJ, Ni L, Witherden IR, Mathieson PW. Direct effects of dexamethasone on human podocytes. Kidney Int (2006) 70(6):1038-45. doi:10.1038/sj.ki.5001655

49. Faul C, Donnelly M, Merscher-Gomez S, Chang YH, Franz S, Delfgaauw J, et al. The actin cytoskeleton of kidney podocytes is a direct target of the antiproteinuric effect of cyclosporine A. Nat Med (2008) 14(9):931-8. doi:10.1038/nm.1857

50. Kreidberg JA, Sariola H, Loring JM, Maeda M, Pelletier J, Housman D, et al. WT-1 is required for early kidney development. Cell (1993) 74(4):679-91. doi:10.1016/0092-8674(93)90515-R

51. Patek CE, Fleming S, Miles CG, Bellamy CO, Ladomery M, Spraggon L, et al. Murine Denys-Drash syndrome: evidence of podocyte de-differentiation and systemic mediation of glomerulosclerosis. Hum Mol Genet (2003) 12(18):2379-94. doi:10.1093/hmg/ddg240

52. Ohtaka A, Ootaka T, Sato H, Soma J, Sato T, Saito T, et al. Significance of early phenotypic change of glomerular podocytes detected by Pax2 in primary focal segmental glomerulosclerosis. Am J Kidney Dis (2002) 39(3):475-85. doi:10.1053/aikd.2002.31391

53. Qin XS, Tsukaguchi H, Shono A, Yamamoto A, Kurihara H, Doi T. Phosphorylation of nephrin triggers its internalization by raft-mediated endocytosis. J Am Soc Nephrol (2009) 20(12):2534-45. doi:10.1681/ASN.2009010011

54. Ruotsalainen V, Ljungberg P, Wartiovaara J, Lenkkeri U, Kestilä M, Jalanko $\mathrm{H}$, et al. Nephrin is specifically located at the slit diaphragm of glomerular podocytes. Proc Natl Acad Sci U S A (1999) 96(14):7962-7. doi:10.1073/pnas. 96.14 .7962
55. Gerke P, Huber TB, Sellin L, Benzing T, Walz G. Homodimerization and heterodimerization of the glomerular podocyte proteins nephrin and NEPH1. JAm Soc Nephrol (2003) 14(4):918-26. doi:10.1097/01.ASN.0000057853.05686.89

56. Akhtar M, Al Mana H. Molecular basis of proteinuria. Adv Anat Pathol (2004) 11(6):304-9. doi:10.1097/01.pap.0000146219.03058.ea

57. Shih NY, Li J, Cotran R, Mundel P, Miner JH, Shaw AS. CD2AP localizes to the slit diaphragm and binds to nephrin via a novel C-terminal domain. Am J Pathol (2001) 159(6):2303-8. doi:10.1016/S0002-9440(10)63080-5

58. Salant J. Podocyte slit-diaphragm protein nephrin is linked to the actin cytoskeleton. Am J Physiol Renal Physiol (2002) 282(4):F585-91.

59. Caridi G, Perfumo F, Ghiggeri GM. NPHS2 (podocin) mutations in nephrotic syndrome. Clinical spectrum and fine mechanisms. Pediatr Res (2005) $57(5 \mathrm{Pt}$ 2):54R-61R. doi:10.1203/01.PDR.0000160446.01907.B1

60. Schwarz K, Simons M, Reiser J, Saleem MA, Faul C, Kriz W, et al. Podocin, a raft-associated component of the glomerular slit diaphragm, interacts with CD2AP and nephrin. J Clin Invest (2001) 108(11):1621-9. doi:10.1172/ JCI200112849

61. Huber TB, Simons M, Hartleben B, Sernetz L, Schmidts M, Gundlach E, et al. Molecular basis of the functional podocin-nephrin complex: mutations in the NPHS2 gene disrupt nephrin targeting to lipid raft microdomains. Hum Mol Genet (2003) 12(24):3397-405. doi:10.1093/hmg/ddg360

62. Karle SM, Uetz B, Ronner V, Glaeser L, Hildebrandt F, Fuchshuber A. Novel mutations in NPHS2 detected in both familial and sporadic steroid-resistant nephrotic syndrome. J Am Soc Nephrol (2002) 13(2):388-93.

63. Huber TB, Hartleben B, Kim J, Schmidts M, Schermer B, Keil A, et al. Nephrin and $\mathrm{CD} 2 \mathrm{AP}$ associate with phosphoinositide 3-OH kinase and stimulate AKTdependent signaling. Mol Cell Biol (2003) 23(14):4917-28. doi:10.1128/MCB. 23.14.4917-4928.2003

64. Dustin ML, Olszowy MW, Holdorf AD, Li J, Bromley S, Desai N, et al. A novel adaptor protein orchestrates receptor patterning and cytoskeletal polarity in T-cell contacts. Cell (1998) 94(5):667-77. doi:10.1016/S0092-8674(00)81608-6

65. Welsch T, Endlich N, Kriz W, Endlich K. CD2AP and p130Cas localize to different F-actin structures in podocytes. Am J Physiol Renal Physiol (2001) 281(4):F769-77.

66. Coppo R. Different targets for treating focal segmental glomerular sclerosis. Contrib Nephrol (2013) 181:84-90. doi:10.1159/000348637

67. Lovatt D, Ruble BK, Lee J, Dueck H, Kim TK, Fisher S, et al. Transcriptome in vivo analysis (TIVA) of spatially defined single cells in live tissue. Nat Methods (2014) 11(2):190-6. doi:10.1038/nmeth.2804

68. Patrakka J, Lahdenkari AT, Koskimies O, Holmberg C, Wartiovaara J, Jalanko $\mathrm{H}$. The number of podocyte slit diaphragms is decreased in minimal change nephrotic syndrome. Pediatr Res (2002) 52(3):349-55. doi:10.1203/00006450200209000-00007

Conflict of Interest Statement: The authors declare that the research was conducted in the absence of any commercial or financial relationships that could be construed as a potential conflict of interest.

Received: 22 July 2014; paper pending published: 28 July 2014; accepted: 01 September 2014; published online: 01 October 2014.

Citation: May CJ, Saleem M and Welsh GI (2014) Podocyte dedifferentiation: a specialized process for a specialized cell. Front. Endocrinol. 5:148. doi: 10.3389/fendo.2014.00148

This article was submitted to Diabetes, a section of the journal Frontiers in Endocrinology.

Copyright (๑) 2014 May, Saleem and Welsh. This is an open-access article distributed under the terms of the Creative Commons Attribution License (CC BY). The use, distribution or reproduction in other forums is permitted, provided the original author(s) or licensor are credited and that the original publication in this journal is cited, in accordance with accepted academic practice. No use, distribution or reproduction is permitted which does not comply with these terms. 